\title{
INSECTICIDAL ACTIVITY OF LOCALLY FORMULATED TAR OIL AS SOLUBLE LIQUID ON THE PINK BOLLWORM, PECTINOPHORA GOSSYPIELLA (SAUNDERS)
}

\author{
AMER, R. A. M. ${ }^{1}$ AND A. G. EL-SISI ${ }^{2}$ \\ 1. Plant Protection Research Institute, ARC, Dokki. Giza \\ 2. Central Agricultural Pesticide Laboratory, ARC, Dokki. Giza
}

(Manuscript received 11 July 2010 )

\begin{abstract}
Crude tar oil was prepared as soluble liquid formulation using wetting and spreading agents alone, acidity modifier alone and mixture of wetting and spreading agents plus acidity modifier in different five formulations, Tar oil (1), Tar oil (2), Tar oil (3), Tar oil (4) and Tar oil (5) in addition of the crude tar oil for controlling the pink bollworm, Pectinophora gossypiella (Saunders) as alternative of conventional pesticides. Toxicity of the prepared tar oils and crude tar oil were determined against egg and newly hatched larvae of the pink bollworm. Latent effects when they used with meduim lethal concentration $\left(\mathrm{LC}_{50}{ }^{\prime} \mathrm{S}\right)$ on the pink bollworm treated as newly hatched larvae which include \% larval mortality, \% pupation, $\%$ moth emergency in addition larval duration, adult longevity, egg laying rate, \% egg hatchability, \% fecundity, \% control of egg hatchability, \% sterility (observed and corrected) compared with untreated. Also their effects on some life table parameters (Speculation) such as The female progeny/female (Mx), Survival rate $(L x)$, net reproductive rate $(R o)$, intrinsic rate of natural increase $\left(r_{m}\right)$, finite rate of increase $\left(e^{r m}\right)$, generation period $(T)$ and doubling time (DT)were studied. The result contains three parts as follows:
\end{abstract}

\section{Toxicity of tar oils against PBW}

Results obtained indicated that tested tar oils especially tar oil (4) was the most toxic formula against eggs since its $\mathrm{LC}_{50}{ }^{\prime} \mathrm{S}$ ranged between $1.481-12.48 \%$ depends on egg age, the 1-day old eggs was the most susceptible compared with other egg ages, followed by tar oil (2) and tar oil (5) while crude tar oil showed the least effect. Also, tests of the efficiency of the tested tar oils against the newly hatched larvae were conducted in two periods, after 1-hour and 3-day later. The results showed that $\mathrm{LC}_{50}$ 'S at 3-day later lower than 1-hour. Tar oil (4) had the most toxicity against newly hatched larvae of the pink bollworm treatments, followed by tar oil (2), tar oil (5), tar oil (3) and tar oil (1), while crude tar oil was the least toxic.

\section{Latent effect of the tested tar oils}

Results indicated that tested tar oils had latent effects similar to insect growth regulator compounds since they caused increase in \%larval mortality, decrease $\%$ pupation and \% moths' emergency. In addition to increase in \% control of egg hatchability and $\%$ sterility (observed and corrected). Opposite, there are decreasing in larval duration, adult longevity especially oviposition period, egg laying rate, \% egg hatchability and \% fecundity. Tar oil (4) showed the highest effect compared with the control. 


\section{Life table parameters}

The female progeny/female $(M x)$ decreased in tested tar oil (4). Survival rate ( $L x)$ decreased. Also, there was a decrease in the net reproductive rate (Ro), increase rate (intrinsic rate of natural increase $\left(r_{m}\right)$ and finite rate of increase $\left(e^{r m}\right)$ ) compared with the control. In contrast, the tested tar oil increased the generation period (T) and doubling time (DT) compared with the control.

\section{INTRODUCTION}

Behavioral and biological studies described that the larvae of the pink bollworm, Pectinophora gossypiella (Saunders) began hatching and continued for 2-3 hour period, newly hatched larvae entered fruiting forms within 30 minutes. Therefore, it could be expected that the proper time of controlling this pest is during egg stage and neonate (Noble, 1969).

High number of chemical pesticides sprays is used for controlling bollworms per season. The result is high input costs, health risks and the presence of pesticide residues in plants often exceeding the maximum residue levels. Pesticide use reduction targets along with requirement for farmers to follow crop specific integrated production guidelines are needed. These facts have simulated considerable interest to mineral and plant oils as alternatives to pesticide. Mineral oils have efficient results against eggs and newly hatched larvae of the Pink bollworm in laboratory and field experiments (Hewady et al., 1993 and Rofail et al. 2000) and also against cotton leafworm (Badr et al., 1995). Khattak \& Rashid (2006) reported that plant neem oil at 1.5 and $2 \%$ reduced the population of spotted bollworms and american bollworms up to 168 hours after spray. In addition, Ratnadass, et al. (2009) showed that physic nut oil (Jatropha curcas) had insecticidal activity against Helicoverpa armigera.

Tar oil that used in this study acts $50 \%$ waste distillation of charcoal manufacture at $550{ }^{\circ} \mathrm{C}$. Tar oils have many toxic compounds as phenols, benzene, sulphate, acids, toluene, perols, aldehide, ...exc. Tar oils is used as a surface treatment method for protection of shingle roofs, boats, fences, ...exc. (Tomlin, 1994) and (Viitanen, et al. 2000).

The biological parameters of the pink bollworm as larval, pupa, adult durations and mortalities...exc. assessed the effect of the tar oils used and gave indication on insect development if it was exposed to the tested compounds. Assessment of the tar oil effects on the fecundity and sterility may through light on their potency.

The speculation (life table parameters) can be used as a guide to investigate the pest population development. This is a valid method for assessment of the efficacy of the compounds used and to clarify the effect of external factors on the growth, 
survival, reproduction, intrinsic and finite rate of increase for the pest population (Abou-Setta, et al., 1986, El- Gemeiy, 2002 and El-Metwally, et al., 2007).

The aim of the present study is preparation the local, cheep and available tar oil as suitable formulation (soluble liquid) and determination its toxic effect, latent effect and speculation (life table parameters) as a new alternative of conventional pesticide against two harmful stages of the pink bollworm, Pectinophora gossypiella (Saunders), the first stage is 1, 2, 3 and 4-day old eggs and the second stage is newly hatched larvae.

\section{MATERIALS AND METHODS}

\section{Materials}

\section{1- The insect}

A laboratory strain of newly hatched larvae and eggs of the pink bollworm, $P$. gossypiella were reared in the Bollworms Department, Plant Protection Research Institute, Agriculture Research Center on a semi artificial diet as described by Rashad and Ammar (1985). Rearing conditions were controlled at $27 \pm 1^{\circ} \mathrm{C}$ and $65-75 \% \mathrm{RH}$.

\section{2- Crude tar oil}

Tar oil, a crude compound from the waste distillation of charcoal manufacture under $550^{\circ} \mathrm{C}$ at Egypt New \& Renewable Energy Authority (NREA), Seventh district, Nasr City. Crude tar oil had the following properties: its free acidity $=6.86 \%$, its surface tension $=42.15 \mathrm{dyne} / \mathrm{cm}$. and soluble in water up to $5 \%$ concentration.

\section{3- Wetting and spreading agent}

Sisi-6: It is anionic surfactant prepared by neutralization of aryl alkyle sulphonic acid with alkaline.

600 Do: It is nonionic surfactant, brief name of polyethylene glycol 600 di-oleate.

400 DL: It is nonionic surfactant, brief name of polyethylene glycol 400 di-laurate.

4- Acidity modifier: $\mathrm{KOH}$ : potassium hydroxide solution $40 \%$ was used as a neutralization material for the acidity of tar oil.

\section{Methods}

\section{I- Preparation of tar oil as soluble liquid formulations}

Crude tar oil compound is prepared in five different formulations as the following:

Tar oil (1) (85\% SL.) contained $85 \%$ tar oil, $10 \%$ Sisi as wetting agent and $5 \%$ water.

Tar oil (2) ( $85 \%$ SL.) contained $85 \%$ tar oil, $15 \% \mathrm{KOH}$.

Tar oil (3) ( $85 \%$ SL.) contained $85 \%$ tar oil, $10 \%$ Sisi $+5 \% \mathrm{KOH}$ solution.

Tar oil (4) (95\% SL.) contained 95\% tar oil + 5\% 600 DO.

Tar oil (5) (95\% SL.) contained 95\% tar oil $+5 \% 400$ DL. 


\section{II- Toxicity of the tested tar oils on the egg stage}

The dipping technique method was used. Three replicates were used for each concentration and each replicate contained number of eggs (1-4) days old ranged between 90 and 150 eggs. The piece of paper contained deposited eggs was dipped for $1 \mathrm{~min}$ in each tested concentrations, another 3 replicates were treated with water for the check. Each treated replicate/each preparation was placed in a clean tube $(3 \times 10 \mathrm{~cm}$.) after water evaporation until hatching under the controlled conditions $\left(27 \pm 1^{\circ} \mathrm{C} \& 65-75 \%\right.$ R.H). The hatched and dead eggs were counted.

\section{III- Toxicity of the tested tar oils on the newly hatched larvae}

Thin film technique was used as a method of application in the present work. Each Petri-dish was treated with $1.0 \mathrm{ml}$ of the tested preparation, five replicates for each preparation and the control were used. The Petri-dish which used as control was treated with water only. Twenty five of newly hatched larvae/replicate was exposed for one hour to the tar oil film in each Petri-dish. The alive larvae from each treatment were counted and transferred singly to clean vials containing the artificial diet and maintained under controlled conditions $\left(27 \pm 1^{\circ} \mathrm{C}\right.$ and $65-75 \%$ R.H.). Then the numbers of alive and dead larvae were counted three days after treatment.

$\mathrm{LC}_{50} \& \mathrm{LC}_{90}$ values were measured by software computer probane. The efficiency of different insecticides could be measured by using Sun 's equation (1950) as follows:

$$
\mathrm{LC}_{50}\left(\mathrm{LC}_{90}\right) \text { of the compound } \mathrm{A}
$$

Toxicity index $=$

$$
\mathrm{LC}_{50}\left(\mathrm{LC}_{90}\right) \text { of the compound } \mathrm{B}
$$

Where A: is the most effective compound.

$\mathrm{B}$ : is the other tested compound.

\section{IV- Latent effect of the tested tar oils}

The pink bollworm, P. gossypiella was treated as newly hatched larvae by $\mathrm{LC}_{50}$ of tar oil formulation, i.e. Tar oil (4) $\left(\mathrm{LC}_{50}=4.278 \%\right)$ and crude tar oil $\left(\mathrm{LC}_{50}=50.98 \%\right)$. The rest alive larvae were fed on artificial diet at $27 \pm 1^{\circ} \mathrm{C}$ and $65-75 \%$ R.H. up to pupal stage, then, the following biological parameters was investigated as follows:

The durations of larvae and pupae surviving treatments/replicate/treatment were recorded. Larval mortality, pupation and moth emergency percentages calculated as follows: 
No. dead larvae

$\%$ Larval mortality

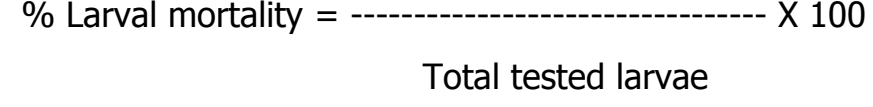

No. produced pupae
\% Pupation = ------------------------------ X 100
Total tested larvae

No. emerged moth
\% Moths emergency = ----------------------------------- X 100
Total tested larvae

Adult longevity was based upon cumulative number of males and females remaining alive each day. Pre-oviposition, oviposition and post-oviposition periods were determined by placing 2-5 pairs of emerged moths in a clean glass cages covered with muslin (17 cm height and 7-12 cm in diameter) till death of adult females.

Egg laying (total number of eggs per female) calculated from daily counts of deposited eggs on piece of paper. Egg hatchability percentage was counted as follows:

No. hatched eggs

$\%$ Egg hatchability $=$ $X 100$

No. deposited eggs

Control of hatchability percentage calculated according to Zidan and AbdelMegeed (1987) as follows:

No. hatched eggs in check - No. hatched eggs in treatment $\%$ Control of hatchability $=$ $X 100$ No. hatched eggs in check

Fecundity percentage calculated according to Crystal and Lachance (1963) as follows:

No. eggs/ treated female $\%$ Fecundity $=$ $X 100$

No. eggs/ untreated female 
Sterility observed percentage calculated according to Zidan and Abdel-Megeed (1987) as follows:

$\%$ Sterility observed $=100-$ Egg hatchability percentage

Corrected sterility percentage calculated according to Zidan and Abdel-Megeed (1987) as follows:

$\%$ Sterility observed - Check

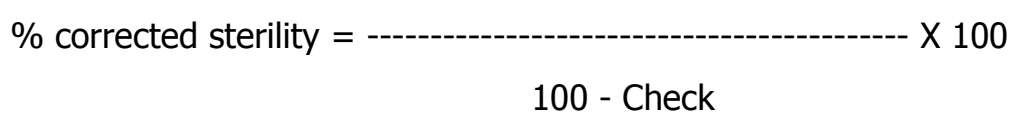

Life cycle extended from egg deposition till adult emergence (days).

All the previous latent effect parameters were statically analyzed using a computer software program costat.

\section{IIV- Speculation (life table) parameters}

The data of speculation (life table) study were analyzed by using life 48 basic computer program of Abou-Setta, et al. (1986).

\section{RESULTS AND DISCUSSION}

\section{A-Toxicity of tested tar oils on the eggs and newly hatched larvae of the pink bollworm}

\section{1- Egg stage}

Table (1) show that tar oil (4) had the most potent against 1-day old eggs $\left(L_{50}=1.481 \%\right)$, followed by tar oil $(2)\left(L_{50}=3.139 \%\right)$, tar oil $(5)\left(L_{50}=6.955 \%\right)$, tar oil (3) $\left(\mathrm{LC}_{50}=9.764 \%\right)$, tar oil $(1)\left(\mathrm{LC}_{50}=10.28 \%\right)$ and crude tar oil $\left(\mathrm{LC}_{50}=\right.$ 93.13\%).

The same trend appeared in 2-day old eggs treatment, $\mathrm{LC}_{50}: 12.48,17.53$, $18.04,18.82,25.16$ and $92.74 \%$ treated by tar oil (4), tar oil (2), tar oil (5), tar oil (3), tar oil (1) and crude tar oil, respectively.

In 3-day old eggs treatments, LC $_{50}: 5.218,8.415,9.184,10.1,11.57$ and $58.78 \%$ when the eggs treated by tar oil (4), tar oil (2), tar oil (5), tar oil (3), tar oil (1) and crude tar oil, respectively.

In 4-day old eggs treatments, $\mathrm{LC}_{50}$ : 11.66, 18.84, 19.05, 24.71, 25.05 and $46.10 \%$ when the eggs treated by for tar oil (4), tar oil (2), tar oil (5), tar oil (3), tar oil (1) and crude tar oil, respectively.

It should be mentioned that one-day old eggs were most susceptible for tar oils treatments. 
Table 1. Toxicity of tested tar oils against the 1, 2, 3 and 4-day old eggs of $P$. gossypiella.

\begin{tabular}{|c|c|c|c|c|}
\hline \multirow{2}{*}{$\begin{array}{l}\text { Tested } \\
\text { Tar oils }\end{array}$} & \multirow{2}{*}{$\begin{array}{c}\mathrm{LC}_{50}(\%) \\
95 \% \text { Confidence limits }\end{array}$} & \multirow{2}{*}{$\begin{array}{c}C_{90}(\%) \\
95 \% \text { Confidence limits }\end{array}$} & \multicolumn{2}{|c|}{ Toxicity index } \\
\hline & & & $\mathbf{L C}_{50}$ & $\mathbf{L C}_{90}$ \\
\hline \multicolumn{5}{|c|}{ 1-day old egg } \\
\hline Tar oil (1) & $\begin{array}{c}10.28 \\
7.369 \pm 12.985\end{array}$ & $\begin{array}{c}95.6 \\
91.99 \pm 99.11\end{array}$ & 14.4 & 13.3 \\
\hline Tar oil (2) & $\begin{array}{c}3.139 \\
1.023 \pm 5.210\end{array}$ & $\begin{array}{c}21.74 \\
18.18 \pm 24.87\end{array}$ & 47.2 & 58.5 \\
\hline Tar oil (3) & $\begin{array}{c}9.764 \\
6.897 \pm 13.12\end{array}$ & $\begin{array}{c}59.18 \\
56.66 \pm 63.22\end{array}$ & 15.2 & 21.5 \\
\hline Tar oil (4) & $\begin{array}{c}1.481 \\
0.822 \pm 3.323\end{array}$ & $\begin{array}{c}12.71 \\
10.88 \pm 15.32\end{array}$ & 100 & 100 \\
\hline Tar oil (5) & $\begin{array}{c}6.955 \\
4.653 \pm 9.951\end{array}$ & $\begin{array}{c}47.165 \\
44.98 \pm 49.99\end{array}$ & 21.3 & 26.9 \\
\hline Crude Tar oil & $\begin{array}{c}93.13 \\
90.82 \pm 97.79\end{array}$ & $\begin{array}{c}1004.7 \\
988.2 \pm 1010.1\end{array}$ & 1.59 & 1.265 \\
\hline \multicolumn{5}{|c|}{ 2-day old egg } \\
\hline Tar oil (1) & $\begin{array}{c}25.16 \\
21.61 \pm 28.88\end{array}$ & $\begin{array}{c}190.3 \\
186.5 \pm 194.4\end{array}$ & 49.6 & 53.8 \\
\hline Tar oil (2) & $\begin{array}{c}17.53 \\
14.03 \pm 19.98\end{array}$ & $\begin{array}{c}109.8 \\
105.5 \pm 113.3\end{array}$ & 71.2 & 93.2 \\
\hline Tar oil (3) & $\begin{array}{c}18.82 \\
15.56 \pm 22.22\end{array}$ & $\begin{array}{c}125.6 \\
122.2 \pm 129.9\end{array}$ & 66.3 & 81.4 \\
\hline Tar oil (4) & $\begin{array}{c}12.48 \\
9.658 \pm 16.61\end{array}$ & $\begin{array}{c}102.3 \\
98.69 \pm 107.8\end{array}$ & 100 & 100 \\
\hline Tar oil (5) & $\begin{array}{c}18.04 \\
14.99 \pm 22.61\end{array}$ & $\begin{array}{c}130.6 \\
127.6 \pm 133.2\end{array}$ & 69.2 & 78.3 \\
\hline Crude Tar oil & $\begin{array}{c}92.74 \\
89.98 \pm 96.96\end{array}$ & $\begin{array}{c}1631.0 \\
1628.3 \pm 1637.1\end{array}$ & 13.5 & 6.27 \\
\hline \multicolumn{5}{|c|}{ 3-day old egg } \\
\hline Tar oil (1) & $\begin{array}{c}11.57 \\
9.874 \pm 14.56\end{array}$ & $\begin{array}{c}181.7 \\
178.8 \pm 189.6\end{array}$ & 45.1 & 64.8 \\
\hline Tar oil (2) & $\begin{array}{c}8.415 \\
6.589 \pm 11.13\end{array}$ & $\begin{array}{c}125.7 \\
121.9 \pm 129.9\end{array}$ & 62.0 & 93.6 \\
\hline Tar oil (3) & $\begin{array}{c}10.1 \\
7.987 \pm 14.49\end{array}$ & $\begin{array}{c}167.6 \\
161.9 \pm 172.9\end{array}$ & 51.7 & 70.2 \\
\hline Tar oil (4) & $\begin{array}{c}5.218 \\
3.256 \pm 8.998\end{array}$ & $\begin{array}{c}117.7 \\
109.6 \pm 122.2\end{array}$ & 100 & 100 \\
\hline Tar oil (5) & $\begin{array}{c}9.184 \\
7.235 \pm 13.32\end{array}$ & $\begin{array}{c}134.7 \\
129.9 \pm 139.9\end{array}$ & 56.8 & 87.4 \\
\hline Crude Tar oil & $\begin{array}{c}58.78 \\
55.55 \pm 64.32\end{array}$ & $\begin{array}{c}908.7 \\
901.9 \pm 914.8\end{array}$ & 8.88 & 12.9 \\
\hline \multicolumn{5}{|c|}{ 4-day old egg } \\
\hline Tar oil (1) & $\begin{array}{c}25.05 \\
21.25 \pm 28.28\end{array}$ & $\begin{array}{c}190.3 \\
184.4 \pm 195.3\end{array}$ & 46.5 & 53.5 \\
\hline Tar oil (2) & $\begin{array}{c}18.84 \\
15.37 \pm 22.58\end{array}$ & $\begin{array}{c}117.6 \\
111.1 \pm 121.6\end{array}$ & 61.9 & 86.6 \\
\hline Tar oil (3) & $\begin{array}{c}24.71 \\
21.21 \pm 27.27\end{array}$ & $\begin{array}{c}142.3 \\
138.5 \pm 149.1\end{array}$ & 47.2 & 71.6 \\
\hline Tar oil (4) & $\begin{array}{c}11.66 \\
8.88 \pm 14.15\end{array}$ & $\begin{array}{c}101.9 \\
98.98 \pm 110.5\end{array}$ & 100 & 100 \\
\hline Tar oil (5) & $\begin{array}{c}19.05 \\
17.02 \pm 22.56\end{array}$ & $\begin{array}{c}186.9 \\
180.2 \pm 192.9\end{array}$ & 61.2 & 54.5 \\
\hline Crude Tar oil & $\begin{array}{c}46.10 \\
41.56 \pm 50.12\end{array}$ & $\begin{array}{c}846.0 \\
839.8 \pm 851.2\end{array}$ & 25.3 & 12.04 \\
\hline
\end{tabular}




\section{2- Newly hatched larvae}

Toxicity of tar oils against the newly hatched larvae of the pink bollworm recorded in two periods, 1-hour and 3-day later treatment shown in table (2). Toxicity for the first period (1-hour later) against the newly hatched larvae, indicated that tar oil (4) showed the most toxic effect followed by tar oil (2), tar oil (5), tar oil (3), tar oil (1) and crude tar oil since the calculated $\mathrm{LC}_{50}$ 's were $10.28,11.87,13.39,15.74,31.8$ and $66.45 \%$, respectively.

At 3-day later, tar oil (4) had the most potent compound against the newly hatched larvae ( $\mathrm{LC}_{50}: 4.278 \%$ ), followed by tar oil (2), tar oil (5), tar oil (3), tar oil (1) and crude tar oil ( $\left(\mathrm{LC}_{50}: 7.760,8.919,9.541,14.22\right.$ and $50.98 \%$ ), respectively. It could be concluded from the aforementioned results that the newly hatched larvae was more susceptible at the second period (3-day later) than the first.

As a general conclusion of toxicity studies for tar oils against both two stages egg and new hatched larvae of pink bollworm, it could be said that tar oil (4) showed the most toxicity against the two stages followed by tar oil (2). The toxicity of tar oil against both eggs and newly hatched larvae of pink bollworm is due to its contents of phenols, creosote and anthrathane (Tomlin, 1994) and Viitanen, et al., 2000).

Table 2. Toxicity of tested tar oils against the newly hatched larvae of $P$. gossypiella.

\begin{tabular}{|c|c|c|c|c|}
\hline \multirow{2}{*}{$\begin{array}{l}\text { Tested } \\
\text { Tar oils } \\
\end{array}$} & \multirow{2}{*}{$\begin{array}{c}\mathrm{LC}_{50}(\%) \\
\text { 95\%Confidence limits }\end{array}$} & \multirow{2}{*}{$\begin{array}{c}\text { LC }{ }_{90}(\%) \\
\text { 95\%Confidence limits }\end{array}$} & \multicolumn{2}{|c|}{ Toxicity index } \\
\hline & & & $\mathbf{L C}_{50}$ & $\mathbf{L C}_{90}$ \\
\hline \multicolumn{5}{|c|}{ One hour later } \\
\hline Tar oil (1) & $\begin{array}{c}31.8 \\
29.09 \pm 36.41 \\
\end{array}$ & $\begin{array}{c}1308.9 \\
1301.3 \pm 1318.9 \\
\end{array}$ & 32.3 & 15.6 \\
\hline Tar oil (2) & $\begin{array}{c}11.87 \\
9.384 \pm 13.83 \\
\end{array}$ & $\begin{array}{c}361.9 \\
359.9 \pm 371.4 \\
\end{array}$ & 86.6 & 56.6 \\
\hline Tar oil (3) & $\begin{array}{c}15.74 \\
14.85 \pm 17.46 \\
\end{array}$ & $\begin{array}{c}380.3 \\
378.9 \pm 386.2\end{array}$ & 65.3 & 53.9 \\
\hline Tar oil (4) & $\begin{array}{c}10.28 \\
9.83 \pm 11.93\end{array}$ & $\begin{array}{c}204.8 \\
199.8 \pm 209.4\end{array}$ & 100 & 100 \\
\hline Tar oil (5) & $\begin{array}{c}13.39 \\
12.89 \pm 15.41 \\
\end{array}$ & $\begin{array}{c}362.1 \\
359.7 \pm 366.6\end{array}$ & 76.8 & 56.6 \\
\hline Crude Tar oil & $\begin{array}{c}66.45 \\
64.32 \pm 68.67 \\
\end{array}$ & $\begin{array}{c}1693.7 \\
1593.7 \pm 1701.6 \\
\end{array}$ & 15.47 & 12.09 \\
\hline \multicolumn{5}{|c|}{ 3-day later } \\
\hline Tar oil (1) & $\begin{array}{c}14.22 \\
11.65 \pm 18.31 \\
\end{array}$ & $\begin{array}{c}980.1 \\
977.7 \pm 986.3 \\
\end{array}$ & 30.1 & 9.18 \\
\hline Tar oil (2) & $\begin{array}{c}7.760 \\
4.998 \pm 10.01 \\
\end{array}$ & $\begin{array}{c}292.0 \\
289.5 \pm 298.5 \\
\end{array}$ & 55.1 & 30.8 \\
\hline Tar oil (3) & $\begin{array}{c}9.541 \\
7.123 \pm 12.54\end{array}$ & $\begin{array}{c}520.8 \\
514.2 \pm 528.6 \\
\end{array}$ & 44.8 & 17.3 \\
\hline Tar oil (4) & $\begin{array}{c}4.278 \\
2.752 \pm 6.929 \\
\end{array}$ & $\begin{array}{c}89.96 \\
85.08 \pm 95.04\end{array}$ & 100 & 100 \\
\hline Tar oil (5) & $\begin{array}{c}8.919 \\
6.154 \pm 11.351\end{array}$ & $\begin{array}{c}336.1 \\
330.3 \pm 341.1\end{array}$ & 47.9 & 26.8 \\
\hline Crude Tar oil & $\begin{array}{c}50.98 \\
46.58 \pm 54.54\end{array}$ & $\begin{array}{c}1514.7 \\
1504.2 \pm 1520.1\end{array}$ & 8.39 & 5.94 \\
\hline
\end{tabular}




\section{B- Latent effect of tested tar oils against the pink bollworm}

The latent effect assessment of the pink bollworm treated as newly hatched larvae by $\mathrm{LC}_{50}$ 'S of tested tar oils described as the following parameters.

\section{1- Larval mortality percentages}

Table (3) showed that larval mortality percent of the pink bollworm treated as newly hatched larvae by $\mathrm{LC}_{50}$ of tar oil (4) at 1-day (initial kill), 3, 5, 7, 9 and 12-day old larvae. The compound gave high larval mortality percent $(72 \%)$ compared with crude tar oil and the control value ( $65 \& 10 \%$, respectively).

Table 3. Latent effect of tested tar oils on larval mortality, pupation and moth emergency of $P$. gossypiella treated as newly hatched larvae.

\begin{tabular}{|c|c|c|c|c|c|c|c|c|c|}
\hline \multirow[b]{2}{*}{$\begin{array}{l}\text { Tested } \\
\text { Tar oils }\end{array}$} & \multicolumn{7}{|c|}{ Larval mortality \% after } & \multirow[b]{2}{*}{$\begin{array}{c}\% \\
\text { Pupation }\end{array}$} & \multirow[b]{2}{*}{$\begin{array}{l}\text { \% Adult } \\
\text { emergency }\end{array}$} \\
\hline & $\begin{array}{l}\text { 1-day } \\
\text { Initial } \\
\text { kill\% }\end{array}$ & $\begin{array}{l}\text { 3- } \\
\text { day }\end{array}$ & $\begin{array}{l}\text { 5- } \\
\text { day }\end{array}$ & $\begin{array}{l}\text { 7- } \\
\text { day }\end{array}$ & $\begin{array}{l}\text { 9- } \\
\text { day }\end{array}$ & $\begin{array}{l}\text { 12- } \\
\text { day }\end{array}$ & $\begin{array}{c}\text { Total } \\
\text { larval } \\
\text { Mortality \% }\end{array}$ & & \\
\hline Tar oil (4) & $20^{\mathrm{a}}$ & $50^{\mathrm{a}}$ & $65^{a}$ & $70^{\mathrm{a}}$ & $71^{\mathrm{a}}$ & $72^{\mathrm{a}}$ & $72^{\mathrm{a}}$ & $28^{\mathrm{c}}$ & $18^{\mathrm{c}}$ \\
\hline $\begin{array}{c}\text { Crude Tar } \\
\text { oil } \\
\end{array}$ & $18^{\mathrm{a}}$ & $50^{\mathrm{a}}$ & $60^{\mathrm{a}}$ & $63^{b}$ & $64^{\mathrm{b}}$ & $65^{b}$ & $65^{\mathrm{b}}$ & $35^{b}$ & $30^{b}$ \\
\hline Control & $0.0^{\mathrm{b}}$ & $3^{b}$ & $6^{\mathrm{b}}$ & $8^{c}$ & $9^{c}$ & $10^{\mathrm{C}}$ & $10^{c}$ & $90^{\mathrm{a}}$ & $85^{\mathrm{a}}$ \\
\hline $\mathrm{F}$ & 84 & 220.9 & 291.9 & 610.4 & 432.4 & 314.5 & 610.4 & 720.7 & 478.6 \\
\hline L.S.D.0.05 & 4.159 & 6.318 & 6.626 & 4.756 & 5.651 & 6.626 & 4.756 & 4.367 & 5.651 \\
\hline
\end{tabular}

Means in the same column followed by the same letter are not significantly different at $p<0.05$.

\section{2- Pupation percentages}

Tar oil (4) is considered effective in decreasing pupation percent (28\%) as illustrated in Table (3), compared with the control value (the pupation is $90 \%$ ).

\section{3- Moth emergency percentages}

As shown in Table (3), tar oil (4) and crude tar oil gave less moth emergency percent. Adult emergency were $18,30 \& 85 \%$, respectively.

\section{4-Larval instars' duration}

When the newly hatched larvae of the pink bollworm treated by tar oils (4) and crude tar oil, larval duration values was less than the control by $1.3-1.4$ days. The fourth larval instars' of the pink bollworm, P. gossypiella had one or two day longer than the second or third instars in the most treatments as shown in Table (4).

\section{5- Pupal duration}

As shown in Table (4), tar oil (4) caused decrease in the pupal duration of the pink bollworm than the control and vice versa, the crude tar oil had increased pupal duration significantly than tar oil (4) and the control. 


\section{6- Adult longevity}

\section{a- adult male longevity}

Table (4) showed that tar oil (4) decreased $9.2 \& 6.57$ days than the tested crude tar oil and control, respectively.

\section{b- adult Female longevity}

The same trend of the tested tar oil (4) and crude tar oil appeared in adult female longevity. Tar oil (4) was effective in decreasing adult female longevity, followed by crude tar oil by 8.94 and 3.6 days, respectively compared with the control as shown in Table (4).

Also, the tested tar oil (4) caused decrease in oviposition period and increase post-oviposition period. In contrast, the oviposition period had long time (13.8 day), while the post-oviposition period had short time (6 days) in the control.

\section{7- Life cycle}

Table (4) showed that tar oil (4) decreased significantly life cycle of pink bollworm (31.54 days), than crude tar oil and the control (35.2 days). In the other hand, crude tar oil increased the life cycle of PBW than the control but in non significant value.

Table 4. Effect of tested tar oils on larval, pupal and adult durations of $P$. gossypiella treated as newly hatched larvae.

\begin{tabular}{|c|c|c|c|c|c|c|c|c|c|c|c|}
\hline \multirow[b]{2}{*}{$\begin{array}{l}\text { Tested } \\
\text { Tar oils }\end{array}$} & \multicolumn{4}{|c|}{ Larval instars' duration } & \multirow[b]{2}{*}{$\begin{array}{c}\text { Pupal } \\
\text { duration }\end{array}$} & \multicolumn{2}{|c|}{ Adult longevity } & \multicolumn{3}{|c|}{ Female adult longevity } & \multirow[b]{2}{*}{$\begin{array}{l}\text { Life } \\
\text { cycle }\end{array}$} \\
\hline & $\begin{array}{c}2^{\text {nd }} \\
\text { instars }\end{array}$ & $\begin{array}{l}3^{\text {rd }} \\
\text { instars }\end{array}$ & $\begin{array}{l}4^{\text {th }} \\
\text { instars }\end{array}$ & Total & & $\sigma^{\pi}$ & 우 & $\begin{array}{c}\text { Pre- } \\
\text { oviposition }\end{array}$ & Oviposition & $\begin{array}{c}\text { Post- } \\
\text { ovipositi } \\
\text { on }\end{array}$ & \\
\hline Tar oil (4) & $6.5^{\mathrm{a}}$ & $6.3^{\mathrm{a}}$ & $8.1^{\mathrm{a}}$ & $20.9^{\mathrm{a}}$ & $6.64^{b}$ & $11.0^{\mathrm{b}}$ & $12.86^{c}$ & $3^{a}$ & $3.86^{\mathrm{b}}$ & $8^{\mathrm{a}}$ & $31.54^{b}$ \\
\hline $\begin{array}{l}\text { Crude } \\
\text { Tar oil }\end{array}$ & $6.4^{\mathrm{a}}$ & $7.2^{\mathrm{a}}$ & $7.4^{\mathrm{a}}$ & $21^{\mathrm{a}}$ & $12.28^{\mathrm{a}}$ & $17.57^{\mathrm{a}}$ & $18.2^{\mathrm{b}}$ & $2^{a}$ & $13.2^{\mathrm{a}}$ & $3^{b}$ & $37.28^{\mathrm{a}}$ \\
\hline Control & $6.7^{\mathrm{a}}$ & $7.4^{\mathrm{a}}$ & $8.2^{\mathrm{a}}$ & $22.3^{\mathrm{a}}$ & $8.9^{b}$ & $20.2^{\mathrm{a}}$ & $21.8^{\mathrm{a}}$ & $2^{\mathrm{a}}$ & $13.8^{\mathrm{a}}$ & $6^{\mathrm{ab}}$ & $35.2^{\mathrm{a}}$ \\
\hline $\mathrm{F}$ & 0.01 & 0.221 & 0.122 & 0.915 & 8.876 & 22.45 & 20.23 & 1.42 & 46.59 & 5.182 & 12.67 \\
\hline L.S.D. 0.05 & 5.286 & 4.316 & 4.315 & 2.825 & 3.329 & 3.460 & 3.460 & 1.998 & 2.825 & 3.826 & 2.825 \\
\hline
\end{tabular}

Means in the same column followed by the same letter are not significantly different at $p<0.05$.

\section{8- Reproductive potential}

Data in Table (5) concerning with eggs of PBW and processes related such as fecundity and hatchability. Female moths resulted from $\mathrm{LC}_{50}$ treated neonate of tested tar oils. Lay reduced significantly number of eggs in comparison to females in control. 
Untreated females produced $189.5 \pm 12$ / female, while, tar oil (4) and crude tar oil produced $116 \pm 6 \& 153.8 \pm 8$ / female, respectively. Tar oil (4) decreased fecundity percentage than crude tar oil.

Also, hatchability percentage affected by previous treatments. The average hatchability percent recorded 89.5, 14.7 \& 89.5\% in control, tar oil (4) \& crude tar oil, respectively (Table 5). Tar oil (4) clearly gave higher control percent of hatchability (90\%) than crude tar oil (48.3\%).

In addition, tar oil (4) was more potent and increased \% sterility than crude tar oil. Statistical analysis proved significant difference between both treatments and untreated (control) (Table 5).

Table 5. Effect of tested tar oils on egg production of $P$. gossypiella treated as newly hatched larvae.

\begin{tabular}{|c|c|c|c|c|c|c|}
\hline $\begin{array}{c}\text { Tested } \\
\text { Tar oils }\end{array}$ & $\begin{array}{c}\text { Egg } \\
\text { Laying } \\
\text { rate }\end{array}$ & $\begin{array}{c}\% \text { Egg } \\
\text { hatchability }\end{array}$ & $\begin{array}{c}\% \text { Control } \\
\text { of } \\
\text { hatchability }\end{array}$ & $\begin{array}{c}\% \\
\text { Fecundity }\end{array}$ & $\begin{array}{c}\% \\
\text { Sterility } \\
\text { observed }\end{array}$ & $\begin{array}{c}\% \\
\text { Corrected } \\
\text { sterility }\end{array}$ \\
\hline Tar oil (4) & $116^{\mathrm{c}}$ & $14.7^{\mathrm{c}}$ & $90^{\mathrm{a}}$ & $61.12^{\mathrm{c}}$ & $85.3^{\mathrm{a}}$ & $83.58^{\mathrm{a}}$ \\
\hline $\begin{array}{c}\text { Crude Tar } \\
\text { oil }\end{array}$ & $153.8^{\mathrm{b}}$ & $57.08^{\mathrm{b}}$ & $48.36^{\mathrm{b}}$ & $81.03^{\mathrm{b}}$ & $42.92^{\mathrm{b}}$ & $36.22^{\mathrm{b}}$ \\
\hline Control & $189.5^{\mathrm{a}}$ & $89.5^{\mathrm{a}}$ & $0.0^{\mathrm{c}}$ & $100^{\mathrm{a}}$ & $10.5^{\mathrm{c}}$ & $0.0^{\mathrm{c}}$ \\
\hline F & 935.3 & 238.9 & 2282.4 & 1700.9 & 1582.9 & 1216.2 \\
\hline L.S.D. 0.05 & 4.159 & 8.397 & 3.263 & 1.631 & 3.263 & 4.159 \\
\hline
\end{tabular}

Means in the same column followed by the same letter are not significantly different at $p<0.05$.

\section{C- Speculation (Life table) parameters}

\section{1- Female progeny/female (Mx) and rate of survival (Lx)}

Figure (1) illustrated that female progeny/female $(M x)$ value of tar oil (4) treatment was near from the control, the values ranged "between" (0.78 to 14.92) and $(0.18$ to 14.84$)$, respectively. The opposite was found in crude tar oil treatment where the $(\mathrm{Mx})$ value was reduced in comparison with the control, it ranged "between" (0.42 to 13.62) female's progeny/female.

The (Lx) parameter (rate of survival) in the same figure ranged "between" (0.29 and 0.93 ) times in the normal females. The females treated as newly hatched larvae with tar oil (4) treatment had the least survival rate that ranged "between" (0.07 and $0.14)$ times compared with the tested crude tar oil $(0.08-0.57)$. 
Figure 1. Effect of tested tar oils on the female progeny/ female (Mx) and survival rate (Lx) of the pink bollworm.
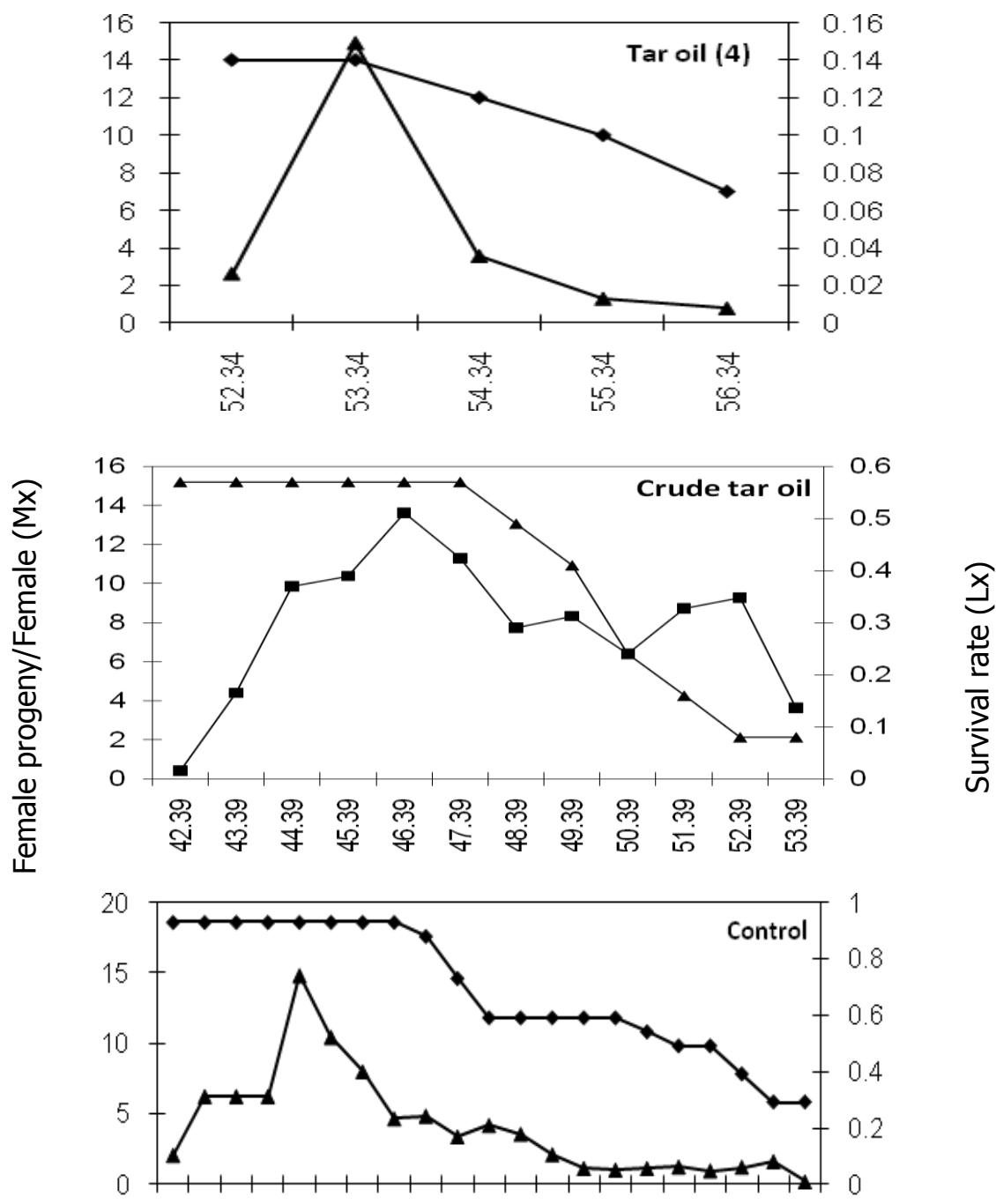

L L L L L L L L L L L L L L L L L L L L L L L L L L L L L L L L L L L L

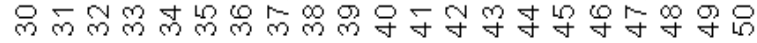

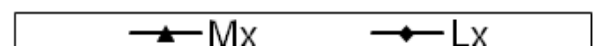

Actual female age $\times$ (days)

\section{2-Generation period $(T)$}

As shown in table (6), the two tested tar oils increased the generation period. Tar oil (4) was 53.50 days and then crude tar oil lasted 46.67 days as compared with that in control (35.35 days).

\section{3- Net reproductive rate (Ro)}

The tested tar oils ( $\mathrm{LC}_{50}$ 's) caused high reduction of net reproductive rate (Ro) when the pink bollworm treated as newly hatched larvae as shown in Table (6). Tested tar oil (4) is considered the reason of the most reduction of net reproductive 
rate to 3.041 females/ female compared with the control ( $\mathrm{Ro}=71.05$ females/female) in one generation, followed by crude tar oil gave the higher increased, 39.75 females/female. Whereas, the two tested tar oils had destructive decreased from net reproductive rate compared with the control data.

\section{4- Increase rate}

\section{1- Intrinsic rate of natural increase $\left(r_{m}\right)$}

Tar oil (4) had the higher reduction of intrinsic rate (0.0208 times/female/day), followed by crude tar oil, the intrinsic rate data was 0.0790 times/female/day compared by intrinsic rate of natural increase $\left(r_{m}\right)$ i.e. the ability of inheriting increase for the normal female was 0.121 times/female/day as shown in Table (6).

\section{2- Finite rate of increase $\left(e^{\mathrm{rm}}\right)$}

The daily population of the normal pink bollworm was 1.128 times/female/day as in Table (6). Also, the females initiated from newly hatched larvae treated by tested tar oils had capacity lower than the control value i.e. tar oil (4) had 1.021 times/female/day, followed by crude tar oil treatment, the finite rates of increase was 1.082 times/female/day.

Table 6. Life table parameters of $P$. gossypiella treated as newly hatched larvae with $\mathrm{LC}_{50}{ }^{\prime} \mathrm{S}$ of tested tar oils.

\begin{tabular}{|c|c|c|c|c|c|c|}
\hline \multirow{2}{*}{ Tested tar oils } & \multirow{2}{*}{$\begin{array}{c}\mathbf{T} \\
\text { (days) }\end{array}$} & \multirow{2}{*}{$\begin{array}{c}\text { Ro } \\
\text { (females/female) }\end{array}$} & \multicolumn{2}{|c|}{$\begin{array}{c}\text { Increase rate } \\
\text { (times/female/day) }\end{array}$} & \multirow{2}{*}{$\begin{array}{c}\text { DT } \\
\text { (days) }\end{array}$} & \multirow{2}{*}{$\begin{array}{l}\text { Sex } \\
\text { ratio }\end{array}$} \\
\hline & & & $r_{m}$ & $e^{r m}$ & & \\
\hline Tar oil (4) & $53.50^{\mathrm{a}}$ & $3.041^{c}$ & $0.0208^{c}$ & $1.021^{\mathrm{c}}$ & $33.32^{\mathrm{a}}$ & $0.78^{\mathrm{a}}$ \\
\hline Crude tar oil & $46.67^{b}$ & $39.75^{\mathrm{b}}$ & $0.0790^{b}$ & $1.082^{\mathrm{b}}$ & $8.774^{\mathrm{b}}$ & $0.45^{c}$ \\
\hline Control & $35.35^{c}$ & $71.05^{\mathrm{a}}$ & $0.121^{\mathrm{a}}$ & $1.128^{\mathrm{a}}$ & $5.747^{b}$ & $0.54^{b}$ \\
\hline $\mathrm{F}$ & 44.49 & 1738.1 & 11269.9 & 4321.5 & 205.8 & 436.5 \\
\hline L.S.D.0.05 & 4.756 & 2.825 & 0.0016 & 0.0028 & 3.648 & 0.028 \\
\hline
\end{tabular}

Means in the same column followed by the same letter are not significantly different at $p<0.05$.
( $\mathrm{T}$ ) $=$ The generation time
(Ro) $=$ The net reproductive rate
$\left(r_{m}\right)=$ The intrinsic rate of natural increase
$\left(e^{r m}\right)=$ The finite rate of increase
(DT) $=$ The doubling time

\section{5- Doubling time (DT)}

The calculated time for population becomes twice that means doubling time (DT) depend on the intrinsic rate of natural increase $\left(r_{m}\right)$ which was affected by many factors as the rate of survival, generation time, female in progeny and fecundity.

The pink bollworm population in control multiplies every 5.747 days (Table 6). This value increased in tar oil treatments to 8.774 and 33.32 days when the pink 
bollworm was treated as newly hatched larvae by crude tar oil and tar oil (4), respectively.

\section{6- Sex ratio}

In control, the sex ratio was 0.54 when the pink bollworm developed from newly hatched larvae. The ratio was increased to 0.78 in case of treatment by tar oil (4) as shown in Table (6). In contrast, in case of crude tar oil treatment the sex ratio decreased to 0.45 compared with the control.

Generally, as described in toxicity action, latent effects and speculation studies, the tested tar oils had satisfaction data to control the pink bollworm, Pectinophora gossypiella (Saunders) in the laboratory. It gives indication to the important usage that the waste liquid (tested tar oils) to control the larval population and infestation of the pink bollworm as a new alternative of pesticide instead of wasting it without using that need more studies on the tar oil application in the fields to ensure the effects of the compound beside to focus on the side effects on the natural enemies, plant phytotoxicity, residual in crop treatment, ...exc.

\section{Acknowledgment}

The authors thank Engineer, Hassan Abdel-Hakim Gomaa, Egypt New \& Renewable Energy Authority (NREA), for his co-operation in preparation of the crude tar oil.

\section{REFERENCES}

1. Abou-Setta, M.M., R.W. Sorrel and C.C. Childers. 1986. Life 48: A basic computer program to calculate life table parameters for an insect or mite species. Florida Entomol. 69 (4): 690-697.

2. Badr, N., A.G. El-Sisi and N. Abdel- Meguid. 1995. Evaluation of some locally for mulated petroleum oils for controlling cotton leafworm, Spodoptera littoralis (Boisd.). J. Agric. Sci. Mansoura Univ., 20 (5): 2557-2563.

3. Crystal, M.M. and L.E. Lachance. 1963. The modification of reproduction in insects treated with alkylating agents. Inhibition of ovarian growth and egg reproduction and hatchability. Biol. Bull., 25: 270-279.

4.

El-

Gemeiy, H.M. 2002. Impact of two formulations of Bacillus thuringiensis var kurstaki on life table parameters of the spiny bollworm Earias insulana (Boisd.). Ann. Agric. Sc. Moshtohor, 40 (3): 1753-1760.

5. El-Metwally, H.E., S.A., El-Mahy, A. Abdel-Hafez and R.A. Amer. 2007. Effect of Some Insecticides on The Life Table Parameters of The Pink Bollworm, Pectinophora gossypiella (Saund.). Egypt.J.Agric.Res., 85(2): 523-534. 
6. El-Sisi, A.G. 1981. Compatibility of some pesticides and growth regulators. M.SC. Thesis, Fac. Agric. Ain Shams Univ.

7. El-Sisi, A.G. and M. El- Hariry. 1991. Formulation and insecticidal efficiency of the Egyptian petroleum oil fractions against cowpea, Aphis craccivora (Koch.). Agric. Res. Rev., 69(1): 297- 305.

8. Hewady, M., A.G. El-Sisi and A. Omar. 1993. Pesticidal efficiency of local petroleum oil fractions against two developmental stages of the bollworms, Pectinophora gossypiella and Earias insulana. Egypt. J. Appl. Sci., 8(7) 494-502.

9. Khan Khattak, M. and M. Rashid. 2006. Evaluation of neem (Azadirachta indica Juss) oil, neem seed water extracts and baythroid TM against bollworms and egg parasitoid, Trichogramma chilonis. Pak. Entomol. Vol. 28, No. 1, 5-10.

10. Noble, L.W. 1969. Fifty years research on the pink bollworm in the united ststes agriculture. Handbook No. 357, Washington, D.C. 20402.

11. Rashad, M.A. and E.D. Ammar. 1985. Mass rearing of the the spiny bollworm, Earias insulana (Boisd.) on semi artificial diet. Bull.Soc.Ent.Egypt, 65: 239-244.

12. Ratnadass, A., M. Togola, B. Cisse and J. Vassal. 2009. Potential of sorghum and physic nut (Jatropha curcas) for management of plant bugs (Hemiptera: Miridae) and cotton bollworm (Helicoverpa armigera) on cotton in an assisted trap-cropping strategy. SAT Journal, V. 7, p: 1-7.

13. Rofail, M., M., Nada, A.G. El-Sisi and A. Rashad. 2000. Time of spraying some natural oils as a limiting factor for controlling cotton bollworm, Pectinophora gossypiella (Saunders). Egypt. J. Agric. Res., 78 (4), 1499-1507.

14. Sun, Y. P. 1950. Toxicity index on improved method of comparing the relative toxicity of insecticides. J. Econ. Entomol., 43: 45-53.

15. Tomlin, C. 1994. The pesticide manual. Incorporating the agrochemical handbook, $10^{\text {th }}$ ed., The Royal society of Chemistry, Crop Protection Publication, pp. 1341.

16. Viitanen, H., A. Hanhijarvi, A. Hukka, and K. Koskela. 2000. Modelling mould growth and decay damages healthy building, design and operation of HVAC. Proceeding. Espoo, 6-10 August Vol.3. 341-346.

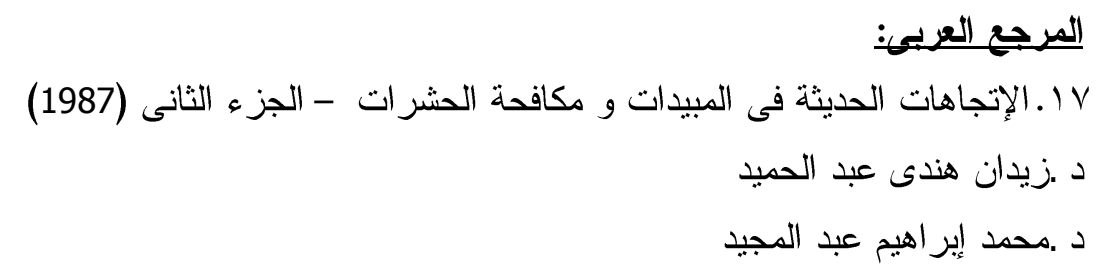




\section{النشاط الإبادى الحشرى للزيت القطرانى المحضر محليا}

\section{على صورة محلول قابل للأوبان على دودة اللوز القرنفلية}

$$
\begin{aligned}
& \text { رضا عبد الجليل محمد عامر } 1 \text { أحمد غازى السيسى2 } \\
& \text { ا ـ ـعه بحوث وقاية النباتات - مركز البحوث الزراعبة - الدقى - جيزة }
\end{aligned}
$$

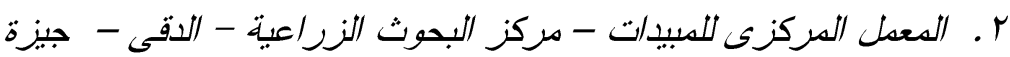

تم تحضير الزيت القطر انى الخام على صورة مستحضر محلول قابل للذوبان باستخدام

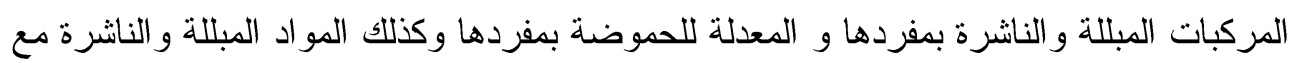

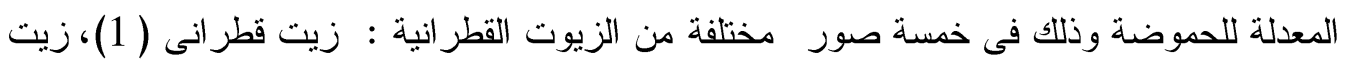

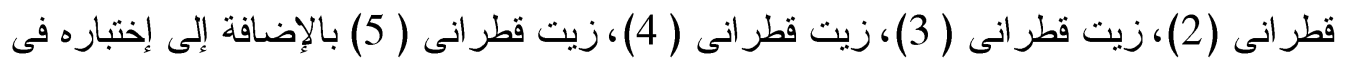
صورته الخام حيث تم تقدير سميتها ضد طورين من الأطوار الضارة لدودة اللوز القرنفلية وهما طور البيض عمر يوم ويومين وثلاثة وأربعة أيام وطور يرقات الفقس الحديث كأحد بدائل المبيدات.

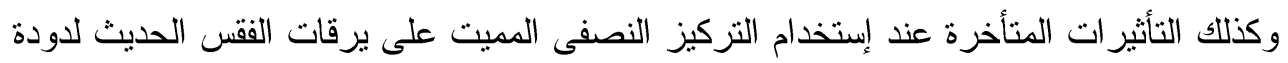

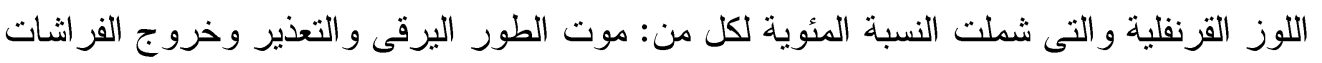

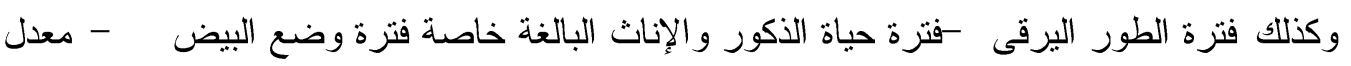
وضع البيض - النسبة المئوية لفقس البيض - الخصوبة - النسبة المئوية لمدى تحكم المركبات فى ولى فئه الفقس - العقم (الظاهرى -المصحح) وذلك مقارنة بقيم الكونترول • بالإضافة إلى دراسة تأثير الزيوت القطر انية على بعض القياسات التنبؤية مثل عدد الإناث الناتجة لكل أنثى (Mx) - معدل البقاء

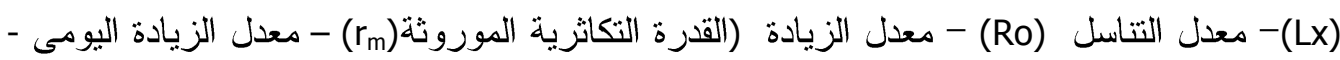
فترة الجيل (T) (e (e)

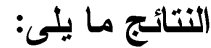

أولا: تقبير السمبة: وجد أن الزيت القطر انى(4) كان أثند الزيوت القطر انية المختبرة سمية حيث تز اوح التزكيز النصفى المميت على البيض بين 1.481 إلى 12.48\% وذلك حسب عمر البيض

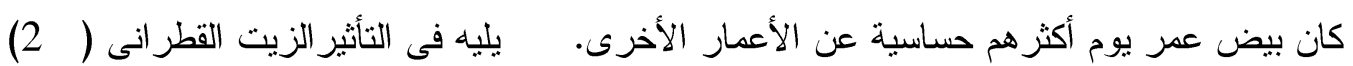
و الزيت القطر اتى (5) بينما كان الزيت القطر انى الخام أقلهم تأثير ا. كما تم إختبار سمية الزيوت القطر انية على طور الفقس الحديث على فترتين: الفترة الأولى بعد لرد مرور ساعة واحدة من المعاملة و الفترة الثانية بعد مرور ثلاثة أيام من المعاملة وقد أظهرت الثيه النتائج

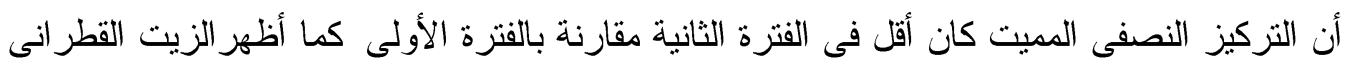
(4) سمية أعلى من الزيوت القطر انية الأخرى على الفقس الحديث لدودة اللوز القرنفلية يليه فى ذلك الكئ

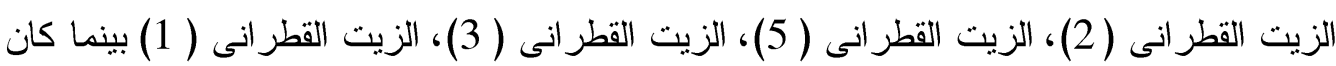
الزيت القطر انى الخام أقلهم سمية مقارنة بالزيوت القطر انية السالفة الذكر. 
ثانبا: التأتثير ات البيولوجية للتركيز النصفى الممبت: ــــم معاملة يرقات الفقس الحديث بالتركيز

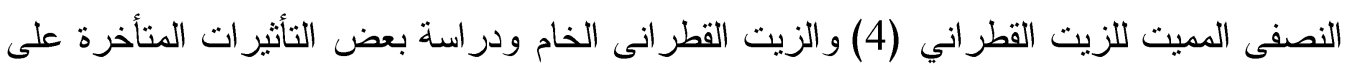

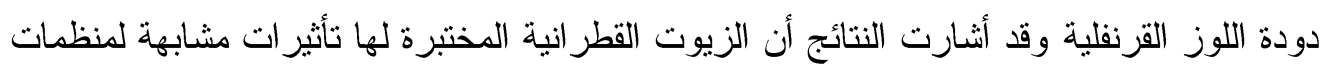

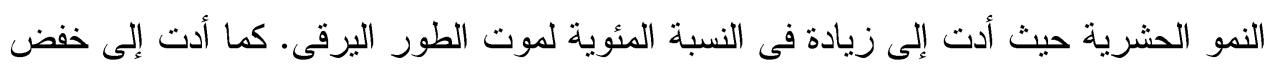
النسبة المئوية للتعذير وخروج الفر اشات - خفض فترة الطور اليرقى - خفض فترة حياة الذكور

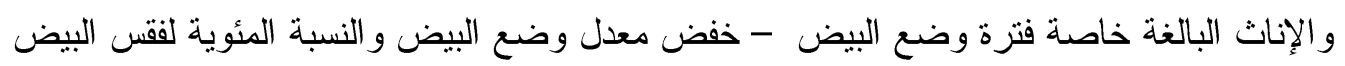
و الخصوبة وتحكم المركبات فى الفقس و العقم (الظاهرى - المصحح).

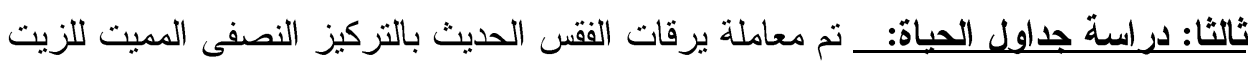

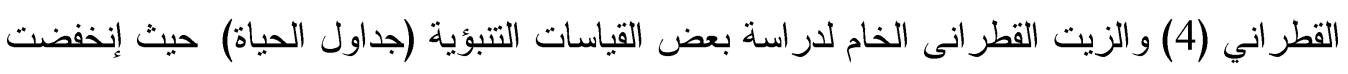

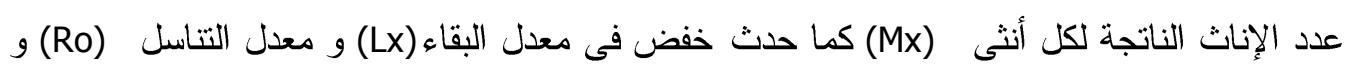

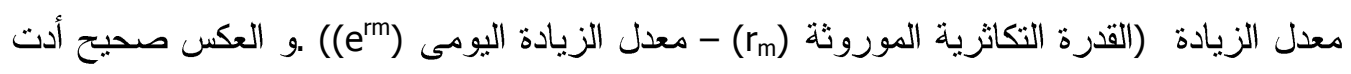

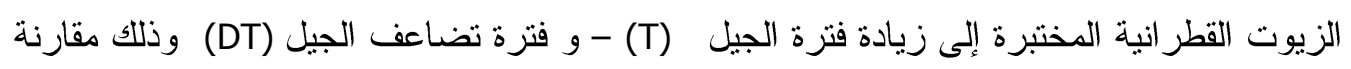
بقيمة الكونترول. 